\title{
El consumo de alcohol en adolescentes: El rol de la comunicación, el funcionamiento familiar, la autoestima y el consumo en la familia y amigos ${ }^{1}$
}

\author{
Gonzalo Musitu Ochoa², Cristian Suárez Relinque², Gonzalo Del Moral Arroyo², María Elena Villarreal \\ González $^{3}$
}

Recibido: 13-03-2015 - Aceptado: 21-05-2015

\begin{abstract}
Resumen
En este estudio se pretende analizar las relaciones existentes entre la comunicación familiar y el funcionamiento y la autoestima (dimensiones familiar y académica) con el consumo de alcohol en adolescentes escolarizados y el consumo de familia y amigos. También, se examinaron estas relaciones en función del sexo. Participaron 1,245 adolescentes mexicanos (50,7\% chicos, edad media de 14,8; 49,3\% chicas, edad media de 14,2) ubicados en centros educativos de secundaria y preparatoria. Se utilizó un muestreo probabilístico estratificado considerando la proporción de alumnos por grado escolar, grupos y turno. Se utilizó el modelo de ecuaciones estructurales en el análisis de los datos. En los resultados, se constató el significativo rol que desempeña la comunicación con los padres y el funcionamiento familiar, a través de la autoestima de protección, en el consumo de alcohol de los adolescentes. También, se observó una relación entre el consumo de familia y amigos y el consumo de alcohol en adolescentes. Finalmente, se obtuvieron diferencias significativas respecto del género.
\end{abstract}

Palabras clave: comunicación, funcionamiento familiar, autoestima de protección, consumo de alcohol.

\section{Alcohol consumption in adolescents: The role of communication, family functioning, self-esteem and consumption in the family and friends}

\begin{abstract}
The aim of the present study was to analyze the relationships between communication in the family and family functioning and self-esteem (dimensions familiar and academic) with the consumption of alcohol in school teenagers and family and friend's consumption. Relationships among variables were also analyzed by gender. Participants in the study were 1,245 Mexican adolescents (50, 7\% male; mean age 14, 8;

${ }^{1}$ Esta investigación se ha elaborado en el marco del Proyecto de Investigación PSI2012-334 "La violencia escolar, de pareja y filio-parental en la adolescencia desde la perspectiva ecológica", subvencionado por el Ministerio Economía y Competitividad del Gobierno de España.

${ }^{2}$ Área de Psicología Social. Universidad Pablo de Olavide. Sevilla, España.

${ }^{3}$ Facultad de Psicología. Universidad Autónoma de Nuevo León. Monterrey, México.
\end{abstract}


BÚSQUEDA - Enero / Junio de 2015 - No. 14 (45 - 61)

and 49, 3\% female; mean age 14.2) drawn from secondary schools. It was used a stratified probability sample method considering students' proportion in school degree, groups and shift. Structural equation model were calculated to analyze the data. Results obtained showed the significant role that plays the communication with parents and the family functioning across the protective self-steem in the consumption of alcohol in adolescents. Also a relation among family and friend's alcohol consumption and adolescent's consumption was observed. Finally differences were obtained in relation to the gender.

Keywords: communication, family functioning, protective self-steem and alcohol consumption.

\section{Introducción}

En el informe de la Organización Mundial de la Salud (OMS, 2008) se afirmaba que el consumo de alcohol constituye una amenaza para la salud pública a pesar de disponer, cada vez más, de información respecto de las consecuencias negativas en la salud social y física, tales como: accidentes de tráfico, altercados con la policía, peleas, asesinatos, urgencias médicas, suicidio, bajo rendimiento escolar (Anderson y Baumberg, 2006; Brown y D'Amico, 2000; Elzo, 2010; Farke y Anderson, 2007; Fernández-Cruz y Marco, 2010; Ministerio de Sanidad, 2010) y daños cerebrales irreversibles (Montesionos y Guerri, 2014). En el caso de la adolescencia, el consumo de alcohol supone uno de los problemas más graves de salud pública debido a que, bajo ciertas condiciones, aumenta la probabilidad de que este consumo se mantenga o agudice durante la vida adulta con los daños y efectos antes señalados, y vinculándose al consumo de otras drogas (Laespada, 2010; Natera, Juárez, Medina, Mora y Tiburcio, 2007; Villarreal, 2006).

La edad de inicio de consumo de alcohol en México se sitúa entre los 13 y 14 años, similar a la de la mayoría de países occidentales (Elzo, 2014; Hernández, 2009; Secretaría de Salud, 2008). Además, según la Encuesta Nacional de Adicciones (ENA, 2011), la prevalencia de consumo de alcohol en adolescentes en México se sitúa en un 26,6\%. Por otra parte, el Estado de Nuevo León se ubica con un $29,7 \%$, cifra que rebasa la media nacional con un $3,1 \%$.

Respecto a las causas de este consumo en la adolescencia, se han analizado variables individuales, familiares, escolares, de los iguales, culturales, comunitarias y de los medios de comunicación (Zamboaga, 2005). En relación con la familia, se ha observado que la comunicación, el afecto y el control parental son variables particularmente importantes para promover una forma de autonomía en los hijos, cimentada en la capacidad de adaptación a las relaciones y a la vida social y, en consecuencia, para prevenir conductas de riesgo (Elzo, 2014; Lila y Gracia, 2005). La importancia de los factores familiares de protección es que limitan y, en ocasiones, bloquean el efecto de los factores de riesgo procedentes de otros entornos, como la presión del grupo de iguales o los modelos mediáticos. Por el contrario, la percepción de carencia de afecto, de aceptación y de apoyo representa un factor crítico que promueve, con frecuencia, el consumo de alcohol cuando el hijo es adolescente (Buelga y Musitu, 2006; Kumpfer, Alvarado y Whiteside, 2003). También, las estrategias parentales autoritarias y negligentes están más presentes entre los adolescentes que consumen alcohol de manera abusiva que las autorizativas e indulgentes (Martínez-Álvarez, Fuertes, Ramos y Hernández-Martín, 2003).

Igualmente, el sentimiento de insatisfacción respecto al sistema familiar y la frustración sentida por el tipo de regulación que imponen los padres, se encuentra asociado a una mayor probabilidad, tanto de inicio en el consumo de alcohol al principio de la adolescencia (Musitu y Pons, 2010; Musitu, Suarez y Del Moral, 2014), como de consumo abusivo a lo largo de todo este período (Abeijón, 2014). En términos generales, estos patrones educativos parentales son más probables de encontrar en los adolescentes que muestran baja autoestima y alta necesidad de afirmación personal (Musitu et al., 2001), 
rasgos que se hallan asociados a una mayor probabilidad de consumo (Azcunaga, 2014).

También, la calidad de la comunicación que tiene lugar en el interior del sistema familiar, una dimensión poco explorada, se ha considerado como muy relevante, al constatar las regularidades que muestra con el consumo de alcohol y otras sustancias (Musitu, Suárez y Del Moral, 2014). Como señalan Martínez-Álvarez et al. (2003), la cohesión familiar y la consistencia entre los padres respecto a la educación de los hijos actúan como factores de protección, ya que promueven en los hijos la autoestima y, por tanto, reducen la vulnerabilidad a la presión grupal. Del mismo modo, la calidad de las relaciones parentofiliales constituye otro factor de protección: una comunicación abierta y positiva, en la que predominan los vínculos emocionales disminuye la probabilidad de consumo de alcohol y drogas en los hijos (Buelga y Pons, 2004; Cava, Murgui y Musitu, 2008; Kumpfer et al., 2003). En general, los adolescentes consumidores abusivos de alcohol perciben a su familia como un contexto conflictivo en el que hay poca comunicación, mientras que los abstemios o los consumidores no abusivos perciben más armonía y comunicación (Elzo, 2010; Musitu y Pons, 2010).

En relación con la autoestima, en este trabajo se ha optado por la perspectiva multidimensional (Shavelson, Hubner y Stanton, 1976; Marsh y O’Neil, 1984). Desde esta perspectiva, la autoestima se define como la valoración que la persona hace de sí misma, de modo que, por un lado, el adolescente puede tener una imagen general de sí mismo favorable o desfavorable, $y$, por otro, puesto que se socializa en diversos contextos como el familiar, escolar o social, también desarrollará una imagen de sí mismo específica en cada uno de ellos (Estévez, Martínez-Ferrer y Musitu, 2006). La autoestima, por tanto, refleja una valoración general o global de uno mismo, así como también valoraciones específicas procedentes de contextos específicos (Cava y Musitu, 2003). La autoestima que procede de los ámbitos familiar y académico opera como factor protector del consumo de alcohol (Jiménez, Musitu, y Murgui, 2008). No obstante, la autoestima del adolescente que procede del ámbito de sus relaciones sociales guarda una relación más compleja con esta conducta. Así, en algunos estudios se ha comprobado que la autoestima social tiene una relación positiva con el consumo juvenil de alcohol, en el sentido de que los adolescentes con alta autoestima social pasan más tiempo con sus iguales que los de baja autoestima y tienen, consecuentemente, más oportunidades de manifestar conductas como fumar o beber que, como bien sabemos, suelen iniciarse en la adolescencia con el grupo de iguales (Wild, Filsher, Bhana y Lombard, 2004). Superado el principio de la adolescencia, los individuos con más facilidad para relacionarse y hacer amigos son, precisamente, los que presentan una mayor probabilidad de consumo, relación que todavía no se ha materializado en la primera adolescencia, durante la cual el deseo de autonomía convive con una mayor supervisión familiar (Cava et al., 2008). En este sentido, es importante considerar que todo aquello que reduce la probabilidad de una conducta de riesgo no debe ser considerado sin más como factor de protección (Musitu y Herrero, 203). Obviamente, no debería prevenirse la autoestima social en los adolescentes, sino más bien potenciarla, aunque canalizándola hacia contenidos congruentes con el cuidado de la salud, al mismo tiempo que se fortalecen otros factores de protección (Jiménez, 2011).

Las autoestimas de protección (familiar y escolar) precisamente se encuentran vinculadas con las relaciones parento-filiales $y$, en consecuencia, la comunicación familiar, las relaciones familiares y la relación del sistema familiar con el consumo de alcohol. En este sentido, el modelado parental de consumo es, igualmente, otro factor familiar que tiene influencia en el consumo de alcohol entre los adolescentes. Así, puede afirmarse que existe una mayor probabilidad de consumo abusivo en los hijos conforme aumenta la frecuencia de consumo alcohólico en sus padres (Buelga et al., 2006; Elzo, 2014 y Marina, 2014). En general, el consumo de alcohol en los padres predispone en los hijos una actitud favorable hacia esta conducta, incluso cuando los padres emiten 
BÚSQUEDA - Enero / Junio de 2015 - No. 14 (45 - 61)

mensajes verbales explícitos en contra de su uso (Varlinskaya, Spear y Spear, 2001). Quiere esto decir que, ante la contradicción entre la conducta de los padres y sus verbalizaciones explícitas, el modelado de comportamientos parentales tendrá un mayor efecto en la conducta de los hijos que las palabras. Naturalmente, el modelado ejerce su influencia no de manera mecánica, sino mediatizada por las interpretaciones que el adolescente haga respecto de la conducta y del modelo, así como por los significados sociales que tenga esa conducta. De este modo, un modelado parental de consumo de alcohol y un mensaje verbal rechazando su consumo representa para el hijo una situación paradójica que pudiera generar en el adolescente una categorización del consumo dentro del estatus de adulto, convirtiéndolo, de este modo, en más atrayente. El modelado de los padres es, en consecuencia, un factor importantísimo para entender el comportamiento de los niños y adolescentes (Bandura, 1999; Musitu et al., 2001). Si un adolescente ha vivido durante 13 o 14 años en un medio en el que ha observado como "normal" el consumo social de determinadas sustancias, entenderá como adecuado que él mismo pueda llevar a cabo esta conducta en su vida social autónoma próxima a estrenarse, que es, justamente, el principio de la adolescencia.

El papel del modelado familiar, por tanto, es decisivo no solo en la génesis del consumo abusivo de alcohol, sino también en la prevención. No se trata de que los padres anulen su consumo de bebidas alcohólicas, sino de ofrecer un modelado de consumo controlado y razonablemente prudente -si es que fuesen consumidores-. Esto es especialmente importante, ya que los adolescentes que beben de manera abusiva no han alcanzado un aprendizaje de la ingesta moderada de esta sustancia, puesto que su consumo ocurre, fundamentalmente, con el grupo de iguales y no con la familia (Budd, Eiser, Morgan y Gammage, 1985; Elzo, 2014). El consumo juvenil de alcohol está vinculado a los momentos de ocio con el grupo de iguales durante los fines de semana e, incluso, los consumidores abusivos no suelen beber entre semana o con su familia (Laespada y Elzo, 2007a). Este dato, sin embargo, no resta importancia a los factores de origen familiar, puesto que los adolescentes expresan en su grupo de iguales las pautas de conducta y pensamiento que han venido adquiriendo durante su vida en familia. Sus rasgos de personalidad, expectativas sociales o prioridades de valor se activan durante la adolescencia, pero ya venían orientadas por la socialización familiar, lo cual, desde una perspectiva socioecológica, ejemplifica un tipo de incidencia mesosistémica, como es la interacción peculiar entre dos microsistemas de influencia, la familia y el grupo de iguales (Melero, 2014).

Teniendo en cuenta estos antecedentes y constatado el interés científico de analizar la autoestima de protección (familiar y académica) desde el modelo multidimensional, muy poco explorado en este ámbito de estudio, junto con el consumo de familia y amigos, y el consumo de alcohol, el objetivo del presente trabajo es estudiar las relaciones familiares (apertura en la comunicación con los padres y funcionamiento familiar) y variables personales (autoestimas de protección -familiar y académica-) con el consumo de alcohol en adolescentes escolarizados, teniendo en cuenta las diferencias en función del sexo (Figura1). De este objetivo general se desprenden los siguientes objetivos específicos: 1) Analizar las relaciones existentes entre las variables familiares y personales junto con el consumo de alcohol de familia y amigos, con el consumo de alcohol en adolescentes escolarizados; y, 2) Analizar las diferencias en las variables del estudio en función del sexo. A partir de estos objetivos se plantean tres hipótesis que se expresan en el modelo teórico propuesto: 1) La apertura de la comunicación y el funcionamiento familiar se relacionan con el consumo de alcohol en adolescentes a través del consumo de familia y amigos; 2) La apertura de la comunicación y el funcionamiento familiar se relacionan con la autoestima de protección y esta con el consumo de alcohol; 3) Existen diferencias en las relaciones planteadas entre chicos y chicas. 


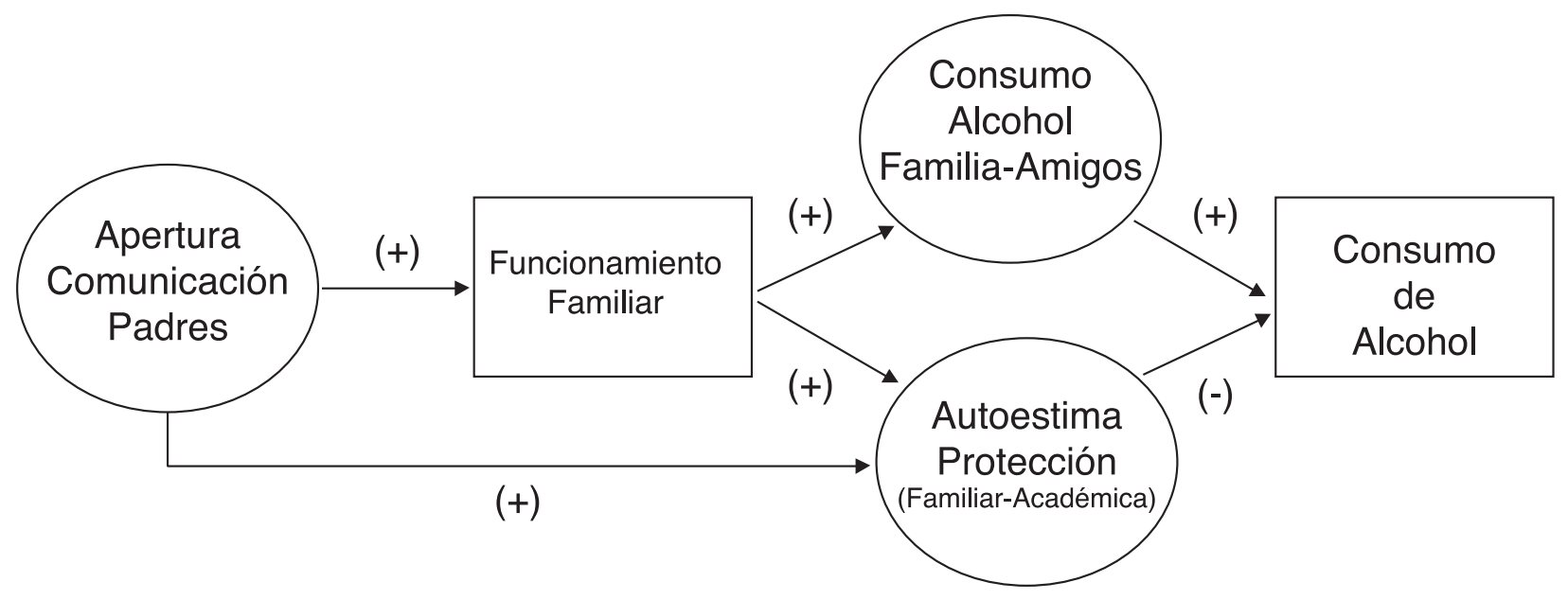

Figura 1. Modelo hipotético de consumo de alcohol

\section{Metodología}

El estudio se realizó mediante un diseño de investigación transversal, ex post facto (Montero y León, 2007) y descriptivo correlacional (Polit y Hungler, 1999).

\section{Participantes}

Se seleccionaron cuatro escuelas públicas de Monterrey, Nuevo León, México y su área metropolitana, dos secundarias y dos preparatorias. La muestra estuvo formada por 1,245 adolescentes escolarizados, con edades comprendidas entre los 12 y los 17 años de edad. La selección de los participantes se realizó mediante un muestreo aleatorio estratificado, considerando la proporción de alumnos por semestre, grupos y turno. Utilizando el programa nQuery Advisor 6.0, estableciendo que el máximo de variables a contemplar para un modelo predictivo serían 20 , con coeficiente de determinación de 0,05 y un poder de 0,90 (Elashoff, 2005). En la tabla 1 se muestran los datos sociodemográficos de la muestra.

\section{Instrumentos}

Cuestionario de ComunicaciónFamiliar(Barnes y Olson, 1982), una adaptación al castellano llevada a cabo por el Grupo Lisis (Facultad de Psicología de la Universidad de Valencia) y
Tabla 1. Características sociodemográficas de la muestra

\begin{tabular}{lrr}
\hline Sexo & & \\
Chicos & 30 & 0,7 \\
Chicas & 15 & 9,3 \\
Edad & & \\
12-14 años (adolescencia temprana) & 55 & 5,4 \\
15-17 años (adolescencia media) & 90 & 4,6 \\
Escolaridad & & \\
Secundaria & 34 & 0,1 \\
Preparatoria & 11 & 9,9 \\
Nivel Socioeconómica & & \\
Baja & 0 & 08 \\
Media Baja & 03 &, 1 \\
Media & 21 & 3,3 \\
Media Alta & 85 & 5,8 \\
Alta & 6 & 02 \\
\hline
\end{tabular}

$\mathrm{N}=1,245$ participantes

adaptada a México por Villarreal, (2009). Es una escala con 20 ítems tipo Likert con cinco opciones de respuesta ( $1=$ nunca a $5=$ siempre). Evalúa la apertura en la comunicación con padre/madre, por ejemplo: «puedo expresarle mis verdaderos 
BÚSQUEDA - Enero / Junio de 2015 - No. 14 (45 - 61)

pensamientos» y problemas en la comunicación padre/madre «hay temas de los que prefiero no hablarle». El coeficiente de fiabilidad (a de Cronbach) para esta muestra fue de 0,86 y 0,87 para padre y madre respectivamente.

\section{Escala de Evaluación Familiar APGAR} (Smilkstein, Ashworth y Montano, 1982). Este instrumento consta de 5 ítems tipo Likert con un rango de respuesta de 0 a 2 ( 0 casi nunca, 1 a veces y 2 casi siempre). Evalúa la cohesión y la adaptabilidad del funcionamiento familiar (e.g., "estás satisfecho(a) con el tiempo que tu familia y tú pasan juntos"). Se establece como disfunción severa una puntuación de 0 a 3 , disfunción moderada de 4 a 6 y como funcionalidad familiar de 7 a 10. El coeficiente de fiabilidad (a de Cronbach) obtenido en su versión original fue de 0,84; y para el presente estudio fue de 0,81.

Cuestionario de Evaluación de la Autoestima en Adolescentes (AFA 5) (García y Musitu, 1999). Este instrumento se compone de 30 ítems tipo Likert con cinco opciones de respuesta (nunca, pocas veces, algunas veces, muchas veces y siempre). Evalúa el autoconcepto de los sujetos en cinco dimensiones: autoestima académica, se refiere a la opinión que tiene el propio individuo de sus aptitudes académicas (e.g., "Mis profesores me consideran un buen estudiante"); autoestima social, hace referencia a la opinión que el propio individuo tiene de sus relaciones sociales (e.g., "Hago fácilmente amigos"); autoestima emocional, alude a la opinión que posee el individuo sobre sus propias emociones (e.g., "Muchas cosas me ponen nervioso"); autoestima familiar, relacionada con la valoración que el propio individuo tiene de sus relaciones familiares (e.g., "Me siento feliz en casa") y, autoestima física, los ítems aluden a la opinión que tiene el sujeto de sus características físicas (e.g., "Me gustan para realizar actividades deportivas"). A mayor puntuación en cada uno de los factores mencionados corresponde mayor autoconcepto en dicho factor. Los coeficientes de fiabilidad obtenidos (Alpha de Cronbach) fueron de 0,86; 0,78; 0,8; 0,78 y 0,79, respectivamente. En este trabajo se utilizaron únicamente los factores familiar y académico.
Consumo de alcohol de la familia y amigos. Se evalúa con dos preguntas directas para conocer el patrón de consumo familiar y de amigos; la primera pregunta se formuló en los siguientes términos: "¿Tus padres y hermanos se emborrachan?"; y la siguiente pregunta hacía referencia al patrón de consumo de los amigos y se redactó de la siguiente manera: “¿Tus amigos se emborrachan?" Las opciones de respuesta iban desde 1, nunca; hasta 5, siempre. A mayor puntuación, mayor consumo de alcohol de familia y amigos.

Escala de Consumo de Alcohol (AUDIT) (Saunders, Aasland, Babor, De La Fuente y Grant, 1993), validado en México por Rubio (1998). Este instrumento se compone de 10 ítems tipo Likert. La pregunta 1 se refiere a la frecuencia del consumo de alcohol y la 2, a la cantidad de consumo. Una alta puntuación en estos dos ítems hace referencia a la frecuencia y cantidad que la persona, en este trabajo, el adolescente, hace del consumo de alcohol. La pregunta 3 es relativa tanto a la frecuencia como a la cantidad en el consumo; este ítem indica que el adolescente bebe más de seis bebidas en una sola ocasión, al menos una vez por semana o diariamente. Las preguntas de la 4 a la 6 indican si existe o no dependencia del consumo de alcohol, y si la persona manifiesta alguno de los siguientes síntomas: no poder parar de beber después de haber iniciado; dejar de hacer algo por beber; beber en la mañana siguiente después de haber bebido en exceso el día anterior, o sentirse culpable o tener remordimientos por haber bebido. Finalmente, las preguntas desde la 7 a la 10 aluden al consumo dañino o perjudicial: el consumidor afirma que se siente culpable por haber bebido; olvidar algo cuando estuvo bebiendo; que se ha lastimado o que alguien ha resultado lesionado como consecuencia de su ingestión de alcohol; y que un amigo, familiar o personal de salud, se ha preocupado por la forma en que bebe. Cada pregunta tiene de tres a cinco posibles respuestas. El punto de corte es 0.8 y cada respuesta tiene un valor numérico que va desde cero hasta dos o cuatro puntos. La sumatoria de las puntuaciones da un puntaje total de 40 puntos como máximo. Los coeficientes de fiabilidad en el presente estudio para cada 
uno de los factores fueron de 0,88; 0,72 y 0,76, respectivamente, los cuales son considerados como aceptables.

\section{Procedimiento}

Una vez seleccionados los centros, el equipo de investigación se reunió con la dirección y profesorado para solicitar los permisos correspondientes y explicarles los objetivos, procedimiento y alcance de la presente investigación. Posteriormente, se solicitó la colaboración voluntaria del alumnado y se les garantizó la confidencialidad y el anonimato de las respuestas y la posibilidad de renunciar a cumplimentar los cuestionarios. Se entregaron también en las escuelas los consentimientos informados para la participación en este trabajo de investigación. Tras la obtención de los permisos correspondientes, se realizó la aplicación del instrumento. Posteriormente, se acordaron las fechas para realizar la administración de los cuestionarios al alumnado. Esta se llevó a cabo por un grupo de investigadores expertos y entrenados. La batería de instrumentos se administró a los adolescentes en sus aulas habituales durante un período regular de clase de aproximadamente 45 minutos. El orden de administración de los instrumentos se contrabalanceó en cada clase y centro educativo. Se informó en todo momento a los adolescentes que la participación en la investigación era voluntaria y confidencial.

\section{Resultados}

Con la finalidad de lograr los objetivos planteados respecto a la relación de las variables familiares y personales con el consumo de alcohol en adolescentes mexicanos, se realizaron los siguientes análisis estadísticos. En primer lugar, se realizó un análisis correlacional de las variables objeto del presente estudio. En la tabla 2, se observa que el consumo de alcohol en los adolescentes correlacionó en sentido negativo con el funcionamiento familiar $(r=-, 074 ; p<, 01)$; con la apertura en la comunicación madre $(r=$ - ,089; $p<, 01)$; la apertura en la comunicación padre $(r=-, 115 ; p<, 01)$; autoestima académica $(r=-152 ; p<, 01)$ y autoestima familiar $(r=-$ ,090; $p<, 01)$; en sentido positivo con el consumo de alcohol de la familia $(r=, 138 ; p<, 01)$ y los amigos $(r=, 298 ; p<, 01)$.

En los resultados se observan correlaciones significativas entre todas las variables, por lo que se incluyeron en el cálculo del modelo de ecuaciones estructurales (Bentler, 1995). Las variables observables y los factores latentes incluidos en el modelo fueron: apertura de la comunicación familiar (indicadores: apertura a la comunicación madre; apertura a la comunicación padre), funcionamiento familiar; autoestima de protección (indicadores: autoestima académica y autoestima familiar); consumo de familia y amigos (indicadores:

Tabla 2. Correlaciones de las variables del modelo

\begin{tabular}{|c|c|c|c|c|c|c|c|}
\hline 1. Consumo de Alcohol & 1 & & & & & & \\
\hline 2. Funcionamiento familiar &, $074^{\star *}$ & & & & & & \\
\hline 3. Apertura a la comunicación madre &, $089^{* *}$ &, $533^{\star *}$ & & & & & \\
\hline 4. Apertura a la comunicación madre &, $115^{\star *}$ &, $492^{* *}$ &, $497^{\star \star}$ & & & & \\
\hline 5. Autoestima académica &, $152^{* *}$ &, $313^{* *}$ &, $340^{* *}$ &, $272^{\star \star}$ & & & \\
\hline 6. Autoestima familiar &, $090^{* *}$ &, $581^{* *}$ &, $529^{\star \star}$ &, $404^{\star *}$ &, $378^{\star *}$ & & \\
\hline 7. Consumo de alcohol familiar &, $138^{\star *}$ &, $160^{\star \star}$ &,$- 098^{\star *}$ &, $070^{\star}$ &, $142^{\star \star}$ &, $125^{\star \star}$ & \\
\hline 8. Consumo de alcohol amigos &, $298^{\star *}$ &, $060^{\star}$ & ,059* & .046 &, $159^{\star \star}$ &, $060^{*}$ &, $180^{\star *}$ \\
\hline
\end{tabular}

**. La correlación es significativa al nivel 0,01 (bilateral)

*. La correlación es significante al nivel 0,05 (bilateral) 
BÚSQUEDA - Enero / Junio de 2015 - No. 14 (45 - 61)

consumo familiar y consumo amigos) y consumo de alcohol. En las tablas 3 y 4 se presentan, para chicos y chicas independientemente, las variables latentes incluidas en el modelo, sus respectivos indicadores, el error estándar y la probabilidad asociada para cada indicador en la variable latente correspondiente. En el caso de las variables observables compuestas por un indicador, la carga factorial toma un valor de $1 \mathrm{y}$ un error de 0 .

\section{CHICOS}

Tabla 3. Estimaciones de parámetros, errores estándar y probabilidad asociada en los chicos.

\begin{tabular}{|c|c|}
\hline \multirow{2}{*}{ Variables } & Carga Factorial \\
\hline & Modelo para chicos \\
\hline \multicolumn{2}{|l|}{ Apertura a la comunicación familiar } \\
\hline Apertura a la comunicación madre & $1 a$ \\
\hline Apertura a la comunicación padre & $1,058 * \star *(0,098)$ \\
\hline \multicolumn{2}{|l|}{ Funcionamiento familiar } \\
\hline Autoestimas de protección & $1 a$ \\
\hline Autoestima académica & $1 a$ \\
\hline Autoestima familiar & $1,491^{\star \star \star}(0,165)$ \\
\hline \multicolumn{2}{|l|}{ Consumo familia y amigos } \\
\hline Consumo familiar & $1 a$ \\
\hline Consumo amigos & $2,034^{* * *}(0,470)$ \\
\hline Consumo de alcohol & $1 a$ \\
\hline
\end{tabular}

\section{CHICAS}

Tabla 4. Estimaciones de parámetros, errores estándar y probabilidad asociada en las chicas

\begin{tabular}{lr}
\hline \multicolumn{1}{c}{ Variables } & \multicolumn{2}{c}{ Carga Factorial } \\
\cline { 2 - 2 } & \multicolumn{2}{c}{ Modelo para chicas } \\
\hline Apertura a la comunicación familiar & $1 \mathrm{a}$ \\
Apertura a la comunicación madre & $1 \mathrm{a}$ \\
Apertura a la comunicación padre & $0,744^{* * *}(0,078)$ \\
$\begin{array}{l}\text { Funcionamiento familiar } \\
\text { Autoestimas de protección }\end{array}$ & $1 \mathrm{a}$ \\
Autoestima académica & \\
Autoestima familiar & $1,703^{* * *}(0,177)$ \\
Consumo familia y amigos & $1 \mathrm{a}$ \\
Consumo familiar & $6,748^{* * *}(1,578)$ \\
Consumo amigos & $1 \mathrm{a}$ \\
Consumo de alcohol &
\end{tabular}

Estadísticos robustos. Error estándar entre paréntesis.

a Fijados en 1,00 durante la estimación.

*** $p<, 001$ (bilateral) 
A continuación, se procedió a contrastar el modelo estimado mediante la técnica de ecuaciones estructurales, utilizando el paquete estadístico EQS 6.1 (Bentler, 1995; Bentler y Wu, 2002; Hu y Bentler, 1999). Se obtuvo un coeficiente de Mardia normalizado de 15,5262, por lo que se utilizaron estimadores robustos, debido a la desviación de la normalidad de los datos. En la tabla 5 se presentan las medidas de bondad de ajuste del modelo estructural de las relaciones entre el consumo de alcohol y las variables seleccionadas, las cuales muestran un ajuste adecuado a los datos [S-B $\chi 2=23,5822$, $g l=14, p<0,05 ; C F I=0,98$, RMSEA $=0,038$ $(0,000-0,064)$. Para el índice CFI se consideran aceptables valores superiores a .95, y para el índice RMSEA, valores inferiores a .05 (Batista y Coenders, 2000). Este modelo explica el $35,9 \%$ de la varianza del consumo de alcohol en adolescentes.

Tabla 5. Medidas de bondad de ajuste del modelo estructural de las relaciones entre el consumo de alcohol y las variables seleccionadas.

\begin{tabular}{lrrr}
\hline Medidas de bondad de ajuste & S-B $\chi 2$ & RMSEA & CFI \\
Valores Obtenidos & 23,5822, gl $=14$ & 0,038 & 0,98 \\
Valores recomendados & & $<0,064$ & $>0,95$ \\
\hline
\end{tabular}

En la figura 2 se muestra la representación gráfica del modelo estructural final con los coeficientes estandarizados y su probabilidad asociada. Los resultados revelaron una relación directa y positiva entre el consumo de familia y amigos y el consumo de alcohol $(\beta=$
$0,581, p<0,001)$. Asimismo, los resultados mostraron relaciones positivas estadísticamente significativas entre la apertura de la comunicación familiar $(\beta=0,662, p<0,001)$ y el funcionamiento familiar ( $\beta=0,340, p<0,001)$, y, de forma negativa y significativa entre la autoestima de protección y

Figura 2. Modelo Explicativo de Consumo de Alcohol

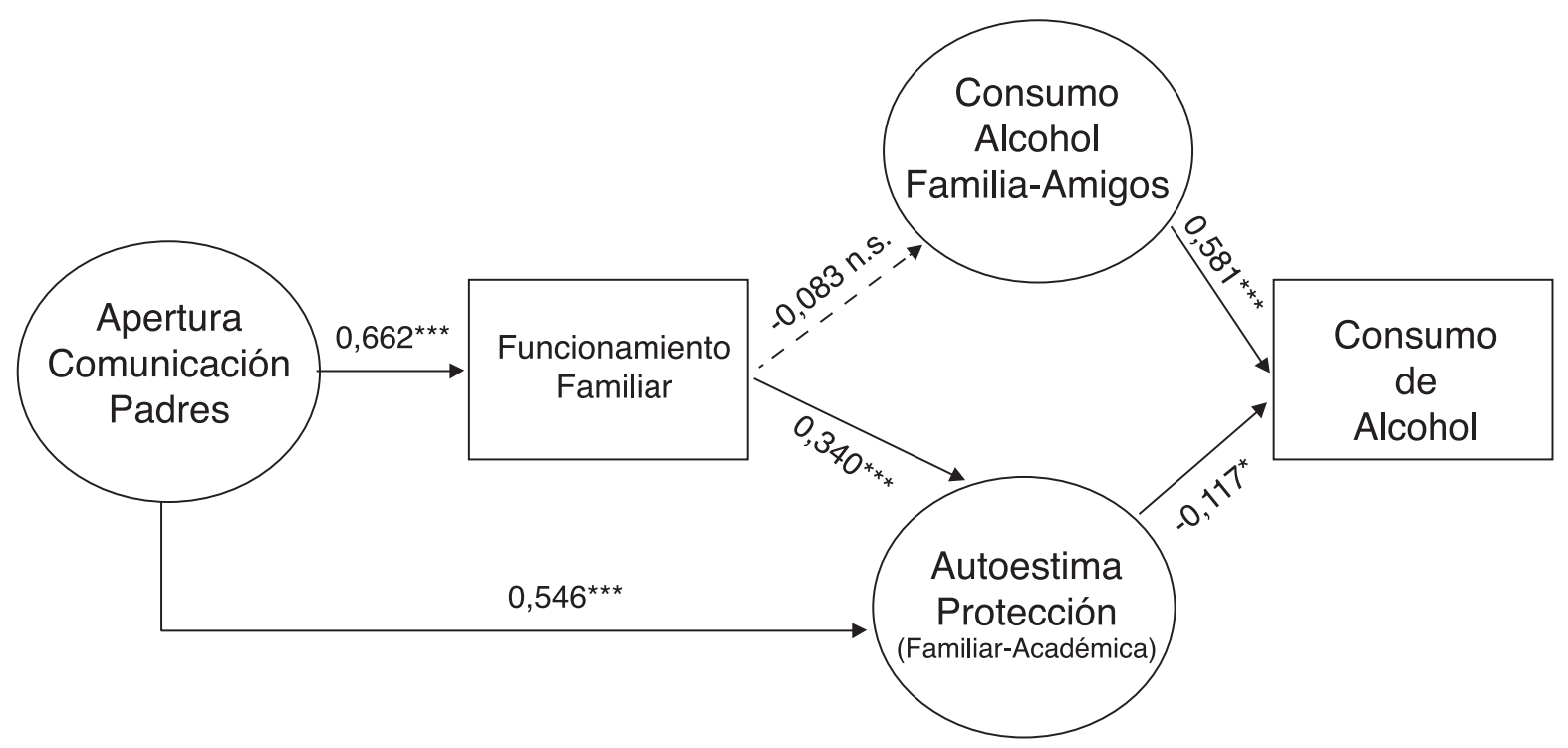

La significación de las relaciones se ha determinado a partir del error estándar ${ }^{* * *} p<0,001 ;{ }^{*} p 0,05$ n.s.0 = no significativo 
BÚSQUEDA - Enero / Junio de 2015 - No. 14 (45 - 61)

el consumo de alcohol. A su vez, se encontró una relación positiva y significativa entre la apertura de la comunicación y la autoestima de protección ( $\beta=0,546, p<0,001) y$, de esta, y de forma negativa, con el consumo de alcohol $(\beta=-0,117$, $p<0,05)$. Finalmente, no se obtuvieron relaciones significativas entre el funcionamiento familiar y el consumo de familia y amigos y el consumo de alcohol ( $\beta=-0,083$, n.s).

\section{Análisis multigrupo del efecto moderador del sexo}

Para dar respuesta al tercer objetivo, se efectuó un análisis multigrupo para comprobar si las relaciones observadas entre las relaciones observadas en el modelo contrastado diferían en función del sexo (Bentler y Wu, 2002). Para llevar a cabo este análisis se estimaron dos modelos. En el primero, las relaciones entre las variables especificadas en la figura 2, fueron estimadas para chicos y chicas sin restricciones (modelo sin restricciones). En el segundo, se calculó un modelo restringido de manera que las relaciones entre las variables eran fijadas como iguales para ambos grupos (chicos y chicas). La diferencia en el valor de la $\chi 2$ entre el modelo restringido y el no restringido fue significativa $\Delta \chi 2(8, N=1277)$ $=34,6474, p<0,001$, lo que indica que el modelo no es equivalente en las relaciones observadas para el grupo de chicos y chicas. Con el fin de determinar que paths o las relaciones, aquí no analizamos las variables generaban estas diferencias, se analizaron los resultados a partir del Test de los Multiplicadores de Lagrange (ML) proporcionado por el EQS. Esta prueba mostró que ambos grupos (chicos y chicas) diferían en dos paths. En primer lugar, respecto a la variable latente apertura a la comunicación padres, se observaron diferencias en la contribución del indicador apertura en la comunicación padre, en el sentido de que la relación fue significativa en ambos sexos pero mayor en el caso de los chicos $(\beta=0,647, p<0,001)$ que en las chicas $(\beta=0,541, p<0,001)$. En el segundo path, se observó que la relación entre el consumo en familia y amigos y la variable final consumo de alcohol fue mayor en los chicos $(\beta=0,498, p<$ $0,001)$ que en las chicas $(\beta=0,293, p<0,001)$. Al liberar estas dos únicas restricciones, el modelo resultó estadísticamente equivalente para ambos grupos $\Delta \chi 2(6, N=1277)=15,8914, p<0,05$.

\section{Conclusiones}

El objetivo del presente trabajo fue estudiar las relaciones entre variables familiares (apertura en la comunicación con padre y madre), funcionamiento familiar y la autoestima de protección (familiar y académica) con el consumo de alcohol en adolescentes escolarizados, teniendo en cuenta las diferencias en función del sexo. Se elaboró un modelo hipotético en el que se expresaron las relaciones entre las variables de estudio. Los resultados obtenidos nos permiten confirmar la mayoría de las relaciones planteadas. Más específicamente, y respecto a la primera hipótesis, "la apertura de la comunicación y el funcionamiento familiar se relacionan con el consumo de alcohol en adolescentes a través del consumo de familia y amigos", se confirma parcialmente, puesto que la relación entre el funcionamiento familiar y el consumo de familia y amigos, en contra de lo esperado, no ha resultado significativo. En el modelo se puede observar cómo la apertura en la comunicación familiar se relaciona de forma directa con el funcionamiento familiar y cómo este no se relaciona con el consumo de familia y amigos. Sin embargo, el consumo de familia y amigos sí se relaciona con el consumo de alcohol.

Es decir, que el consumo de alcohol es independiente del funcionamiento familiar, porque lo que realmente es significativo es el consumo de la familia y los amigos, y este consumo, puede tener lugar en situaciones de buen o mal funcionamiento familiar. Otro aspecto muy distinto sería si se analizase el consumo a través del conflicto familiar, en cuyo caso es muy probable, de acuerdo con otros estudios (Sanz et al. 2004; Kaplan, 2001) que el consumo diferiría de las familias que no experimentan conflictos. Respecto a los amigos, es bien conocido que son un referente social relevante para la ingesta de alcohol, en la medida en que el consumo se incrementa cuando se está en grupo (Hernanz, 2014). Se podría afirmar, junto con numerosos 
autores, que el contacto con los amigos estimula la bebida compulsiva con mucha más frecuencia que en situaciones de soledad (Henry, Slater y Oetting, 2005; Talbott et al., 2008; Villarreal y Landero, 2008; Villarreal et al., 2010). Podría ser de interés en otros trabajos analizar el efecto del funcionamiento de la familia en la elección de los amigos para el disfrute del ocio y del tiempo libre que, se supone, se relacionaría con los modelos no muy bien tratados todavía en relación con la selección de los iguales: el modelo de la compensación -se busca en los iguales lo que no se encuentra en la familia-, y el modelo de la mutua potenciación -se busca en los iguales aquello que se encuentra en la familia- (Fuligni y Eccles, 1993).

Respecto a la segunda hipótesis, "la apertura de la comunicación y el funcionamiento familiar se relacionan con la autoestima de protección y esta con el consumo de alcohol", se confirma plenamente. Lo que se infiere del modelo contrastado es el rol significativo que cumple la comunicación con los padres en la potenciación de las autoestimas de protección -familiar y académica-, y en el funcionamiento familiar que, a su vez, también contribuye a una mayor autoestima de protección y, en función de nuestros resultados, a un menor consumo de alcohol. Estos vienen a confirmar la idea de que la comunicación y el funcionamiento familiar son parte esencial en la vida de los niños y adolescentes, en la medida en que activan procesos que potencian recursos como la autoestima de protección, que es clave en la vida emocional y en la regulación de la conducta. Este resultado se considera relevante en la medida en que son pocos los trabajos en los que se explora el rol de la autoestima de protección desde una perspectiva multidimensional.

Este rol de la autoestima de protección en el consumo de alcohol es también coincidente con los resultados de trabajos recientes (SánchezSosa, Villarreal-González, Ávila y Vera, 2014), e indirectamente con los resultados de otros trabajos en los que también se ha subrayado la importancia de esta autoestima en la regulación de la conducta en niños y adolescentes en otros aspectos de su vida y, en consecuencia, la importancia de potenciar este recurso en programas de intervención para que niños y adolescentes tengan una mayor capacidad de regular su conducta (Marina, 2014). En este sentido, en el modelo calculado, se puede observar que la apertura de la comunicación con los padres tiene una alta relación con la autoestima de protección, la cual, como ya se ha comentado anteriormente, tiene una relación negativa con el consumo de alcohol (VillarrealGonzález, Sánchez-Sosa y Musitu, 2013).

De nuestros resultados se infiere, por una parte, la importancia de la familia en la potenciación de recursos, en este caso la autoestima de protección, y la relevancia de esta en la regulación de la conducta como es el consumo de alcohol en adolescentes y, por otra, el interés para los investigadores de adoptar la multidimensionalidad de la autoestima en la medida en que se está comprobando que hay un efecto diferencial de sus dimensiones respecto al ajuste en los adolescentes. Cabe destacar que los resultados de diversas investigaciones (Jiménez, 2006; Jiménez et al., 2008) apoyan la tesis de que no existe un efecto homogéneo y protector de los distintos tipos de autoestima frente a la implicación del adolescente en problemas de carácter externalizante como el consumo de alcohol, sino que el efecto de las diferentes dimensiones difiere en función del tipo de problema estudiado. En cualquier caso, se confirma que las autoestimas familiar y académica son factores de protección frente a las conductas de riesgo. Por otra parte, Brendgen et al. (2004) aludían a la idea de que había un "lado oscuro" de la autoestima y que, por ejemplo, una autoestima muy elevada en determinadas dimensiones (social y física) podría suponer un mayor riesgo de implicarse en problemas de carácter externalizante como la conducta disruptiva y el consumo de alcohol. No obstante, este es un aspecto en el que se considera se debería continuar profundizando por sus implicaciones prácticas, fundamentalmente, en los programas de prevención. 
BÚSQUEDA - Enero / Junio de 2015 - No. 14 (45 - 61)

En síntesis, los resultados obtenidos, matizan la idea clásica que los investigadores tienen de la autoestima, al considerarla como un recurso psicológico que necesariamente potencia el ajuste saludable del adolescente (Harter, 1990; Taylor y Brown, 1994). En este sentido, es importante tener en cuenta que el consumo de alcohol y otras sustancias durante la adolescencia es una conducta fundamentalmente social y que el consumo moderado $u$ ocasional es relativamente normativo en el contexto cultural mexicano y en otros contextos culturales (Elzo, 2014; ENA, 2011 y Villarreal-González, 2009). Es decir, el consumo de alcohol, agrade o no, es un elemento necesario e instrumental en el desarrollo del adolescente. El consumo de alcohol es rito y provocación a la vez. Es rito porque, normalmente, se consume con tanta naturalidad y asunción dentro de las pautas culturales que no necesita sostenerse en otras justificaciones, pero también es provocación porque en una sociedad donde los espacios formales se encuentran tan predeterminados en los lugares donde las seguridades se anteponen al posible riesgo, a los jóvenes se les deja poco espacio para la experimentación, para asumir sus propios riesgos, para provocar a la sociedad, para marcar distancia con lo que les indican los adultos que es una parte esencial en la construcción de la identidad en adolescentes. El alcohol, como afirma Laespada (2010), "tiene una funcionalidad innegable entre la población adolescente de lo permitido y no permitido dentro de las posibilidades configuradas socialmente..." (p.33).

De este modo, parece factible y legítimo pensar que los adolescentes que consumen alcohol con la familia y con los iguales se autoperciban como "personas sociales normales" e incluso que se autoevalúen positivamente en los ámbitos personal y familiar y, lo más Ilamativo, es probablemente el sentimiento que tienen de que no están cometiendo ninguna infracción. A partir de los resultados de este trabajo creemos que uno de los grandes desafíos en la investigación respecto al consumo de alcohol es diferenciar con claridad qué adolescentes de los que beben alcohol lo están haciendo de manera crónica y quienes de manera experimental y, a poder ser, con estudios longitudinales.
Respecto a la tercera hipótesis, "existen diferencias en las relaciones planteadas entre chicos y chicas" se confirma en parte en la medida en que se han observado diferencias significativas solamente en las dimensiones apertura a la comunicación padres y su indicador apertura a la comunicación padre, el consumo en familia y amigos y el consumo de alcohol. En relación con la comunicación, los resultados son muy sugerentes en la medida en que, además de ser muy pocos los trabajos en los que se incida en esta problemática, matiza aspectos respecto de un proceso en el que padres e hijos están inmersos, porque la comunicación es el medio a través del cual su relación puede desarrollarse y cambiar hacia una mayor mutualidad y reciprocidad. La comunicación es, en palabras de Noack y Krake (1998), "el motor de la transformación de las relaciones entre padres e hijos" (p.67), lo cual implica que no es solo un aspecto más que cambia durante la adolescencia de los hijos, sino que, sobre todo, comienza a reconocerse como un aspecto o dimensión fundamental en la transformación de dicha relación.

En este sentido, y aunque chicos y chicas no difieren en la comunicación que mantienen con la madre, sí se han encontrado diferencias en la comunicación con el padre. En concreto, los chicos manifiestan una mayor apertura en la comunicación con el padre y una menor percepción de problemas en dicha comunicación. Estos resultados, que podrían ser explicados en función de posibles diferencias de género en la socialización familiar o en la asignación de roles paternos y maternos y, obviamente, de variables culturales, resaltan la necesidad de profundizar en el análisis de estas diferencias todavía poco exploradas. Igualmente, sería también muy sugerente ampliar esta investigación incluyendo la percepción que los padres tienen de la comunicación con sus hijos e hijas.

También se ha observado, a partir del análisis multigrupo, que la relación entre el consumo de familia y amigos y el consumo de alcohol es mayor en chicos que en chicas, lo cual podría explicarse por el hecho de que los chicos son 
más consumidores de alcohol que las chicas, con la salvedad de que año tras año se va igualando el patrón de consumo en ambos sexos (chicos: 59,29\%; y chicas: 40,71\%) (Nash, González, Hernández y Bravo, 2012). También, estos resultados se pueden atribuir al hecho de que el consumo de alcohol ha estado más presente en los escenarios de ocio en los chicos que en las chicas (Elzo, 2010, Musitu y Pons, 2010).

Finalmente, este trabajo proporciona observaciones sugerentes y relevantes sobre ciertas variables familiares, sociales y personales que intervienen en el consumo de alcohol en los adolescentes. Se ha observado que la familia puede convertirse, tanto en fuente destacada de factores de riesgo, como de factores de protección y, en consecuencia, su papel esencial en la prevención, que debe considerarse desde la promoción de la salud y contemplarse dentro de los procesos de socialización y comunicación en la familia, los cuales solo son un reflejo de su nivel de funcionamiento. Ante el consumo de alcohol juvenil, los padres suelen asumir una de estas dos posturas: autocrítica y asunción de su responsabilidad como educadores; o de manifiesta adhesión al modelo cultural de consumo de alcohol como lubricante social, lo cual genera un cierto estado de confusión, al tiempo que reconocen su falta de preparación para asumir la responsabilidad educativopreventiva. Los padres deben ser conscientes de la influencia que su comportamiento ejerce en el desarrollo de sus hijos y en sus conductas y deben conocer todo aquello que ejerce un impacto en el ajuste psicológico y social.

En todo caso, no hay que pensar que un adolescente educado en familias con buenas prácticas de potenciación de recursos se convertirá en inmune ante los ofrecimientos del consumo de alcohol y sus efectos. No puede afirmarse esto, de ninguna manera. Es necesario recordar que todo factor de riesgo o de protección actúa conjuntamente con otros factores y tal actuación es solo ponderable en términos de probabilidad. Si bien los factores familiares de protección reducen la probabilidad de consumo abusivo, existen otros factores de índole sociocultural a los que se hallan expuestos todos por ser miembros de la misma sociedad y que creemos de interés seguir explorando en otros trabajos. Realmente, se podría afirmar que la socialización familiar fundamentada en la comunicación y dimensiones relacionadas, es un factor de protección, como también lo es el modelado de consumo moderado o la abstinencia en los padres; pero no solo la familia es responsable de la educación de los menores, también las instituciones sociales tienen su responsabilidad en la prevención y, en general, en la tarea educativa. Sin embargo, es importante reseñar que los resultados expuestos aquí deben interpretarse con cautela, debido a la naturaleza transversal y correlacional de los datos que, como es bien sabido, no permite establecer relaciones causales entre las variables. Un estudio longitudinal con medidas en distintos tiempos ayudaría a la profundización de las relaciones aquí observadas. Pese a estas limitaciones, creemos que este trabajo puede efectivamente orientar a quienes diseñan programas de prevención e intervención en el ámbito de la adolescencia y, concretamente, en el consumo de alcohol y otras sustancias.

\section{Referencias bibliográficas}

Abeijón, J. A. (2014). Como tratar desde la clínica el consumo de alcohol. En T. La espada, El consumo de alcohol entre adolescentes y jóvenes (pp. 168-184). Bilbao: Universidad de Deusto.

Anderson, P. y Baumberg, B. (2006). Alcohol in Europe: A public health perspective. Londres: Institute of Alcohol Studies.

Azcunaga, B. (2014). La atención sanitaria urgente en casos de intoxicación etílica con menores. En T. Laespada (Ed.), El consumo de alcohol entre adolescentes y jóvenes (pp. 23-44). Bilbao: Universidad de Deusto.

Bandura, A. (1999). A sociocognitive analysis of substance abuse: An agentic perspective. Psychological Science, 10, 214-217. 
BÚSQUEDA - Enero / Junio de 2015 - No. 14 (45 - 61)

Barnes, H. y Olson, D. H. (1982). Parent adolescent communication scale. En D. H. Olson, H. McCubbin, H. Barnes, A. Larsen, M. Muxen, y W. Wilson (Eds.), Family Inventories: Inventories Used in a National Survey of Families across the Family Life Cycle (pp. 33-48). St. Paul: University of Minnesota Press.

Batista, J. M., y Coenders, G. (2000). Modelos de ecuaciones estructurales. Madrid: La Muralla.

Bentler, P. M. y Wu, E. (2002). EQS 6 for windows user's guide Multivariate Software, Encino, CA.

Bentler, P. M. (1995). EQS structural equations program manual. Encino, CA: Multivariate Software.

Brendgen, M., Vitaro, F., Poulin, F., Wanner, B. y Turgeon, L. (2004). Is there a dark side of positive illusions? Overestimation of social competence and subsequent adjustment in aggressive and nonaggressive children. Journal of Abnormal Child Psychology, 32 (3), 305-320.

Brown, S. A. y D'Amico, E.J. (2000). Facilitating adolescent self-change for alcohol problems: A multiple brief intervention approach. Documento presentado en 108th Annual Convention of the American Psychological Association. Washington, EEUU.

Budd, R. J., Eiser, J., Morgan, M. y Gammage, P. (1985). The personal characteristics and life-style of the young drinker: The results of a survey of British adolescents. Drug and Alcohol Dependence, 16, 145-157.

Buelga, S. y Musitu, G. (2006). Famille et adolescence: Prévention de conduites à risque. En D. Jacquet, M. Zabalia y $\mathrm{H}$. Lehalle (Eds.), Adolescences d'aujourd'hui. Rennes: Presses Universitaires.
Buelga, S. y Pons, J. (2004). Alcohol y adolescencia: ¿Cuál es el papel de la familia? Encuentros en Psicología Social, 2, 39-43.

Buelga, S., Ravenna, M., Musitu, G. y Lila, M. (2006). Epidemiology and psychosocial risk factors associated with adolescents' drug consumption. En S. Jackson y L. Goossens (Eds.), Handbook of adolescent development. Hove: Psychology Press.

Cava, M. J. y Musitu, G. (2003). La potenciación de la autoestima en la escuela. Barcelona: Paidós.

Cava, M. J., Murgui, S. y Musitu, G. (2008). Diferencias en factores de protección del consumo de sustancias en la adolescencia temprana y media. Psicothema, 20, 389395.

Elashoff, J. (2005). nQuery Advisor Version 6.0. User's guide. Los Angeles, CA: Statistical Solutions Ltd.

Elzo, J. (2010). ¿Hay un modelo mediterráneo de consumo de alcohol? En J. Elzo (Coord.), Hablemos de alcohol (pp.47-67). Madrid: Entimema.

Elzo, J. (2014). La necesidad de comprender el alcohol en adolescentes y jóvenes. En T. Laespada (Ed.), El consumo de alcohol entre adolescentes y jóvenes (pp. 136157). Bilbao: Universidad de Deusto.

Encuesta Nacional de Adicciones. (2011). Consejo Nacional contra las adicciones. México.

Estévez, E., Martínez-Ferrer, B., y Musitu, G. (2006). La autoestima en adolescentes agresores y víctimas en la escuela: La perspectiva multidimensional. Intervención Psicosocial, 15, 223-232.

Farke, W., y Anderson, P. (2007). Binge drinking in Europe. Adicciones, 19, 333-340. 
Fernández-Cruz, A. y Marco, J. (2010). Dimensión médica de los consumos de alcohol en los menores españoles. En J. Elzo (Coord.), Hablemos de alcohol (pp. 115-135). Madrid: Entimema.

Fuligni, A.J. y Eccles, J.S. (1993, julio-noviembre). Las relaciones entre padres e hijos percibidos y orientación temprana de los adolescentes hacia sus compañeros. Psicología del Desarrollo, 29, 622-32.

García, F. y Musitu, G. (1999). Autoconcepto Forma 5. Madrid: TEA.

Harter, S. (1990). Issues in the assessment of the self-concept of children and adolescents. En A.M. La Greca (Ed.), Through the eyes of the child: Obtaining self-reports from children and adolescents (pp. 292-325). Boston: Allyn y Bacon.

Henry, K., Slater, M. y Oetting, E. (2005). Alcohol Use in Early Adolescence: The Effect of Changes in Risk Taking, Perceived Harm and Friends' Alcohol Use. Journal of Studies on Alcohol and Drugs 66, 275-283.

Hernández, T. (2009). La edad de inicio en el consumo de drogas, un indicador del consumo problemático. Intervención Psicosocial, 18, 199-212.

Hernanz, M. (2014). ¿Y si el adolescente no bebe? En T. Laespada (Ed.), El consumo de alcohol entre adolescentes y jóvenes (pp. 174-191). Bilbao: Universidad de Deusto.

Hu, L. y Bentler, P. M. (1999). Cutoff criteria for fit indexes in covariance structure analysis: Conventional criteria versus new alternatives. Structural Equation Modeling, 6, 1-55.

Jiménez, T. I. (2006). Familia y Problemas de Desajuste en la Adolescencia: el Papel Mediador de los Recursos Psicosociales. Universidad de Valencia (Tesis Doctoral).
Disponible en: http://www.uv.es/lisis/otraspublica/tesis_terebel.pdf

Jiménez, T. I. (2011). Autoestima de Riesgo y Protección: Una Mediación entre el Clima Familiar y el Consumo de Sustancias en Adolescentes. Psychosocial Intervention, 20 (1), 53-61.

Jiménez, T. I., Musitu, G. y Murgui, S. (2008). Funcionamiento familiar y consumo de sustancias en adolescentes: El rol mediador de la autoestima. Internacional Journal of Clinic and Health Psychology, 8, 139-151.

Kaplan, J. A. (2001). Family relationships and parent-child alliances: Their role in shaping the connections between parents' marriage and children's adaptations. Dissertation Abstracts International: Section B: the Sciences y Engineering, 61(7-B), 3848.

Kumpfer, K. L., Alvarado, R. y Whiteside, H. (2003). Family-based interventions for substance use and misuse prevention. Substance Use and Misuse, 38, 1759-1787.

Laespada, M. T. (2010). La dimensión sincrónica del deber en la España de hoy. Los menores como punto de especial atención y protección social ante el alcohol. En J. Elzo (Coord.), Hablemos de alcohol (pp. 13-34). Madrid: Entimema.

Laespada, T. y Elzo, J. (2007a). Consumo de alcohol de los adolescentes: Hablando de cifras y datos. En E. Megías (Ed.), Adolescentes ante el alcohol. Barcelona: Fundació La Caixa, 36-57.

Lila, M. y Gracia, E. (2005). Determinantes de la aceptación-rechazo parental. Psicothema, 15, 301-319.

Marina, J. A. (2010). Programas educativos para la prevención del abuso de alcohol En J. Elzo (Coord.), Hablemos de alcohol (pp. 93-114). Madrid: Entimema. 
BÚSQUEDA - Enero / Junio de 2015 - No. 14 (45 - 61)

Marsh, H. W. y O’Neil, R. (1984). Self Description Questionnaire III (SDQ III): The construct validity of multidimensional self-concept ratings by late-adolescents. Journal of Educational Measurement, 21, 153-174.

Martínez-Álvarez, J. L., Fuertes, A., Ramos, M. y Hernández-Martín, A. (2003). Consumo de drogas en la adolescencia: Importancia del afecto y de la supervisión parental. Psicothema, 15, 161-16.

Melero, J. C. (2014). Hackeando el desarrollo de las drogodependencias: del foco en las substancias al desarrollo personal. En T. Laespada (Ed.), El consumo de alcohol entre adolescentes y jóvenes (pp. 97-114). Bilbao: Universidad de Deusto.

Ministerio de Sanidad (2010). Encuesta Domiciliaria sobre Consumo de Alcohol y Drogas 2009- 2010. Madrid, Ministerio de Sanidad.

Montero, I. y León, O. (2007). Guía para nombrar los estudios de investigación en psicología. International Journal of Clinical and Health Psychology, 7, 847-862.

Montesinos y Guerri (2014). Consecuencias neuropatológicas y conductuales del abuso de alcohol durante la adolescencia. En T. Laespada (Ed.), El consumo de alcohol entre adolescentes y jóvenes (pp. 145-168). Bilbao: Universidad de Deusto.

Musitu, G. y Herrero, J. (2003). El rol de la autoestima en el consumo moderado de drogas en la adolescencia. Sociotam: Revista Internacional de Ciencias Sociales, 13, 285-306.

Musitu, G. y Pons, J. (2010). Adolescencia y Alcohol: Buscando significados en la persona, la familia y la sociedad. En J. Elzo (Coord.), Hablemos de alcohol (pp. 13-34). Madrid: Entimema.
Musitu, G., Buelga, S., Lila, M. y Cava, M.J. (2001). Familia y adolescencia. Madrid: Síntesis.

Musitu, G., Suarez, C. y del Moral, G. (2014). Reflexiones en torno al consumo de alcohol en adolescentes. En T. Laespada (Ed.), El consumo de alcohol entre adolescentes y jóvenes (pp. 80-96). Bilbao: Universidad de Deusto.

Nash, N., González, J., Hernández, L. S., Bravo, L. A. y López, M. F. (2012). Prevalencia en el consumo de alcohol en los adolescentes. PsicologiaCientifica.com, 14(13). En:http:// www.psicologiacientifica.com/prevalenciaconsumo-alcohol-adolescentes.

Natera, G., Juárez, F., Medina, M. E. y Tiburcio M. (2007). Alcohol and drug consumption, depressive features, and family violence as associated with complaints to the Prosecutor's Office in Central Mexico. Substance Use and Misuse 42, 1485-1504.

Noack, P. y Krake, B. (1998). Continuity and change in family interactions across adolescence. En M. Hofer, J. Younisy.

Noack, P. (Eds.), Verbal interaction and development in families with adolescents (pp. 65-81). London: Ablex Publishing.

Organización Mundial de la Salud (OMS 2008). Informe sobre la salud en el mundo 2004. Recuperado el 21 noviembre, 2010, de http://www. who.int/whr/2004/es/

Polit, D. F. y Hungler, B.P. (1999). Nursing Research: Principles and Methods (6th edn). Philadelphia: J.B. Lippincott.

Rubio, G. (1998). Validación de la prueba para la identificación de trastornos por el uso de alcohol (AUDIT) en atención primaria. Revista Clínica Especializada, 198, 11-14.

Sánchez-Sosa, J. C., Villarreal-González, M.E., Ávila, M. E. y Vera, A. (2014). Contextos de consumo de drogas ilegales en 
adolescentes escolarizados. Psychosocial Intervention, 23 (1), 69-78.

Sanz, M. et al. (2004). El conflicto parental y el consumo de drogas en los hijos y las hijas. Bilbao: AEFFA

Saunders, J., Aasland O., Babor T., De La Fuente J. y Grant, M. (1993). Development of the Alcohol Use Disorders Identification Test (AUDIT): WHO collaborative project on early detection of persons with harmful alcohol consumption-II 88: 791-804.

Shavelson, R. J., Hubner, J. J. y Stanton, G. C. (1976). Validation of construct interpretations. Review of Educational Research, 46, 407-441.

Smilkstein, G., Ashworth, C. y Montano, D. (1982). Validity and reliability of the Family APGAR as a test of family function. Journal Family Practising, 15, 303-11.

Talbott, L. L., Martin, R. J., Usdan, S. L., Leeper, J. D., Umstattd, M.R., Cremeens, J. L. y Geiger, B. F. (2008). Drinking likelihood alcohol problems, and peer influence among first-year college students. The American Journal of Drug and Alcohol Abuse, 34, 433-440.

Taylor, S. E. y Brown, J. D. (1994). Illusion and wellbeing: A social psychological perspective on mental health. Psychological Bulletin, 103, 193-210.

Varlinskaya, E. I., Spear, L. P. y Spear, N.E. (2001). Acute effects of ethanol on behavior of adolescents rats: Role of social context. Alcoholism Clinical and Experimental Research, 25, 377-385.

Villarreal, M. (2006). Predictores en el consumo de alcohol en estudiantes de preparatoria. Tesis de Maestría. Facultad de Psicología, Universidad Autónoma de Nuevo León. México
Villarreal, M. E., Sánchez-Sosa, J. C. Musitu, G. y Varela, R. (2010). El consumo de alcohol en adolescentes escolarizados: Propuesta de un modelo sociocomunitario. Intervención Psicosocial, 19, 253-264.

Villarreal-González, M. y Landero, R. (2008). La relación de las variables sociodemográficas en el consumo de alcohol en estudiantes de preparatoria. En Moral, J., Landero, R. y González, M. (Eds.), Psicología de la salud en adolescentes y jóvenes. Monterrey. Facultad de Psicología, Universidad Autónoma de Nuevo León.

Villarreal- González, M., Sánchez-Sosa, J.C. y Musitu, G. (2013). Análisis psicosocial del consumo de alcohol en adolescentes mexicanos. Revista Universitas Psychologica, 12(3), 15-31.

Villarreal-González, M. E. (2009). Un Modelo Estructural del Consumo de Drogas y Conducta Violenta en Adolescentes Escolarizados. Tesis Doctoral (Dirs. R. Landero y G. Musitu). Universidad Autónoma de Nuevo León. México.

Wild, L. G., Filsher, A. J., Bhana, A. y Lombard, C. (2004). Associations among adolescent risk behaviours and self-esteem in six domains. Journal of Child Psychology and Psychiatry, 45, 1454-1467.

World Health Organization (2010). European Status Report on Alcohol and Health. Copenhague: WHO Regional Office for Europe.

Zamboaga, B. L. (2005). Alcohol expectancies and drinking behaviors in Mexican American college students. Addictive Behaviors, 30, 673-684. 